\title{
Estimaciones empíricas de mortalidad natural en el bacalao de profundidad (Dissostichus eleginoides Smitt, 1898)
}

\author{
Empirical natural mortality estimates for toothfish (Dissostichus eleginoides Smitt, 1898) \\ Luis Cubillos ${ }^{1}$ y Miguel Araya ${ }^{2,3}$ \\ ${ }^{1}$ Laboratorio de Evaluación de Poblaciones Marinas (EPOMAR), Departamento de Oceanografía \\ Facultad de Ciencias Naturales y Oceanográficas, Universidad de Concepción \\ Casilla 160-C, Concepción, Chile \\ ${ }^{2}$ Departamento de Ciencias del Mar, Universidad Arturo Prat, Casilla 121, Iquique, Chile \\ ${ }^{3}$ Dirección actual: Programa de Doctorado en Ciencias Aplicadas, mención Sistemas Marinos Costeros, \\ Facultad Recursos del Mar, Universidad de Antofagasta, Casilla 179, Antofagasta, Chile \\ lucubillos@udec.cl
}

\begin{abstract}
The instantaneous natural mortality rate is estimated for toothfish (Dissostichus eleginoides) using empirical models, with the emphasis on an evaluation of the precision of the estimates. The results are compared with estimates obtained per age classes and obtained through an analytical model. The growth parameters and the size at first maturity available for toothfish are used in order to consider the error in the input parameters, and after the prediction error in the variance computation of the estimates. The $M$ values were estimated with low precision, with coefficients of variation ranging between 41 y $60 \%$, and confidence intervals

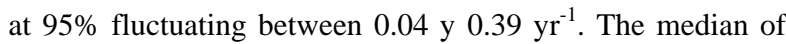
the estimates ranged between 0.1 and $0.14 \mathrm{yr}^{-1}$, and they were closed to the average of the $M$ values obtained per age classes $\left(M=0.11 \mathrm{yr}^{-1}\right)$. It is recommended to use $M=0.1 \mathrm{yr}^{-1}$ for stock assessment models applied to the resource, since centesimal values make no sense for an imprecise parameter.
\end{abstract}

Key words: Empirical models, precision, life history parameters, fisheries
Resumen.- Se estima la tasa instantánea de mortalidad natural $(M)$ en el bacalao de profundidad (Dissostichus eleginoides) utilizando métodos empíricos, con énfasis en la evaluación de la precisión de los estimados. Los resultados se comparan con estimaciones por clases de edad obtenidas por un método analítico. Se utilizan parámetros de crecimiento y talla media de madurez sexual disponible para el recurso con el objeto de considerar el error en los parámetros de entrada, para luego incorporar el error de predicción en la determinación de la varianza de los estimados. Las estimaciones de $M$ fueron poco precisas, con coeficientes de variación que fluctuaron entre 41 y $60 \%$, y límites de confianza de $95 \%$ que fluctuaron entre 0,04 y $0,39 \mathrm{año}^{-1}$. La mediana fluctúo entre 0,1 y 0,14 año $^{-1}$, y fueron comparables con el valor promedio por clases de edad $\left(M=0,11\right.$ año $\left.^{-1}\right)$. Se recomienda utilizar $M=0,1$ año $^{-1}$ en modelos de evaluación del recurso, ya que no tiene sentido utilizar centésima para un parámetro poco preciso.

Palabras clave: Modelos empíricos, precisión, parámetros de historia de vida, pesquerías

\section{Introducción}

En la dinámica de poblaciones explotadas, la tasa de mortalidad natural $(M)$ está relacionada con todas las posibles fuentes de mortalidad, excepto la pesca. Usualmente, valores de $M$ se pueden estimar mediante la aplicación de uno o varios de los siguientes métodos: (a) análisis de curvas de captura para stock levemente explotados (Robson \& Chapman 1961, Ricker 1975), (b) mediante la relación entre la tasa de mortalidad total y el esfuerzo de pesca (Beverton \& Holt 1957,
Paloheimo 1980), (c) técnicas de estimación directa, usualmente costosas y limitadas (i.e. marcaje); y (d) parámetros de la historia de vida y variables ambientales utilizando modelos empíricos o relaciones (Beverton 1963, Rickhter \& Efanov 1976, Pauly 1980, Hoening 1983). Entre estos métodos, los modelos empíricos son los más utilizados para determinar la tasa de mortalidad natural, ya que demandan poca información. Sin embargo, se debe considerar que las estimaciones de $M$ siempre estarán sujetas a una gran incertidumbre (Vetter 1988). 
En Chile, el bacalao de profundidad (Dissostichus eleginoides Smitt, 1898) comenzó a ser explotado comercialmente en la década de los años 70 por una flota artesanal en la zona central. La actividad pesquera que se sustenta en este recurso se desarrolló rápidamente, extendiendo su operación hacia el norte y principalmente hacia el sur, donde los rendimientos fueron mayores (Oyarzún et al. 2003a, b). En la actualidad, la flota artesanal opera con puertos base en Iquique, Antofagasta, Caldera, San Antonio, Constitución, San Vicente, Lebu, Valdivia, y Quellón (Young et al. 1997). Uno de los parámetros más importante para evaluar la situación del stock de bacalao de profundidad lo constituye la tasa de mortalidad natural $(M)$. Al respecto, Yánez \& Pizarro (1984) utilizan $M=0,1$ y 0,2 año $^{-1}$, sobre la base de estimaciones realizadas con métodos empíricos. Al sur de la latitud $47^{\circ} \mathrm{S}$, Zuleta \& Moreno (1992) estiman $M=0,15$ año $^{-1}$, a partir de datos de captura por edad. En el Atlántico Sur, Shust et al. (1990) presentan valores de 0,16 y 0,18 año ${ }^{-1}$ para la población de las islas Georgia del Sur, Moreno \& Rubilar (1992) estimaron $M=0,14 \mathrm{año}^{-1} \pm 0,03$, en tanto Arana et al. (1994) comunican valores de $M$ entre 0,1 y 0,2 año $^{-1}$, indicando que el valor más probable es de 0,11 año- $^{-1}$. En las islas Malvinas, Des Clers et al. (1996) utilizan $M=0,1$ año $^{-1}$ para evaluar el stock de bacalao de profundidad.

Si bien los valores de $M$ que se han utilizado para el bacalao de profundidad caen en el rango comprendido entre 0,1 y 0,2 año $^{1}$, se debe considerar que, por lo general, las estimaciones de $M$ son muy poco precisas cuando se utilizan métodos empíricos, particularmente debido al error de predicción (Pascual \& Iribarne 1993, Cubillos et al. 1999). Para el caso del bacalao de profundidad no se ha evaluado la precisión de las estimaciones de $M$ utilizando métodos empíricos, siendo altamente probables que los valores en el rango $0,1-0,2$ año $^{-1}$ no sean estadísticamente diferentes. En este trabajo, se evalúa la precisión de las estimaciones de $M$ considerando el error en los parámetros de entrada y el error de predicción siguiendo la metodología utilizada por Cubillos et al. (1999), consistente en estimar la varianza de $M$ utilizando el método Monte Carlo. A su vez, se analizan cambios edad-específicos en $M$ con el objeto de comparar el rango de grupos de edad en los cuales podrían aplicarse las estimaciones de $M$ con métodos empíricos.

\section{Material y métodos}

\section{Métodos empíricos}

La aplicación de los métodos se basó en Cubillos et al. (1999), considerando las fuentes de incertidumbre asociada a los datos de entrada a los modelos empíricos, así como el error de predicción que proviene de cada modelo. Se utilizaron los métodos de Rickhter \& Efanov (1976), Pauly (1980), Hoening (1983) y el de Alverson \& Carney (1975) modificado por Cubillos (2003) (Tabla 1).

Tabla 1

Modelos empíricos para la estimación de la tasa instantánea de mortalidad natural

Empirical models for instantaneous natural mortality rate estimation

\begin{tabular}{|c|c|c|}
\hline Método & Modelo & Definición de parámetros y unidades \\
\hline $\begin{array}{l}\text { Rickhter \& Efanov } \\
\text { (1976) }\end{array}$ & $M=\left(\frac{1,521}{T_{m}^{0,72}}\right)-0,155$ & $T_{m}=$ edad de madurez sexual (años) \\
\hline Pauly (1980) & $\begin{array}{l}\log _{10} M=-0,0066-0,270 \log _{10} L_{\infty} \\
\quad+0,6543 \log _{10} K+0,4634 \log _{10} T\end{array}$ & $\begin{array}{l}L_{\infty}=\text { longitud asintótica }(\mathrm{cm}, \mathrm{LT}) \\
K=\text { coeficiente de crecimiento }\left(\mathrm{año}^{-1}\right) \\
T=\text { temperatura promedio anual del hábitat }\left({ }^{\circ} \mathrm{C}\right)\end{array}$ \\
\hline Hoening (1983) & $\ln (Z)=1,44-0,982 \cdot \ln \left(T_{\max }\right)$ & $\begin{array}{l}Z=\text { tasa de mortalidad natural }(\approx M) \\
T_{\max }=\text { longevidad observada (años) }\end{array}$ \\
\hline $\begin{array}{l}\text { Alverson \& Carney } \\
\text { (1975) modificado por } \\
\text { Cubillos (2003) }\end{array}$ & $M=\frac{3 K(1-\bar{\omega})}{\bar{\omega}}$ & $\begin{array}{l}\bar{\omega}=0,62=\text { razón entre la talla crítica y la } \\
\text { longitud asintótica } \\
K=\text { coeficiente de crecimiento }\left(\mathrm{año}^{-1}\right) \text { del modelo } \\
\text { de von Bertalanffy }\end{array}$ \\
\hline
\end{tabular}


El método de Rickhter \& Efanov (1976) expresa una relación entre $M$ (mortalidad natural) y la edad media de madurez sexual ( $T_{\mathrm{m}}$, criterio $50 \%$ ). La talla de primera madurez sexual del bacalao de profundidad fluctúa entre 80 y $115 \mathrm{~cm}$ de longitud en el caso de las hembras, mientras que en los machos podría ocurrir entre los 75 y 100 cm (Oyarzún et al. 2003a, b). Young et al. (1999) señalan que en la mayoría de los casos, la maduración de las hembras ocurre entre los 90 y $100 \mathrm{~cm}$ citando a Zhivov \& Krivoruchko (1990). Oyarzún et al. (2003a) estimaron la talla de primera madurez sexual de machos en 86,7 cm de longitud, mientras que en hembras fluctúo entre 113 y $117 \mathrm{~cm}$. La talla de primera madurez sexual corresponde a la longitud en que el 50\% de las hembras está madura. En este estudio se utilizó una distribución uniforme entre 80 y $117 \mathrm{~cm}$ para estimar la mortalidad natural con el método de Rickhter \& Efanov (1976). Una vez obtenida la longitud de madurez, se calculó la edad promedio de madurez con la ecuación de crecimiento inversa de von Bertalanffy, i.e.

$$
T_{m}=t_{0}-\frac{1}{K} \ln \left(1-\frac{L_{m}}{L_{\infty}}\right)
$$

donde $t_{0}, K$, y $L_{\infty}$ son los parámetros de crecimiento del modelo de von Bertalanffy, los cuales se obtuvieron de la literatura (Tabla 2).
Pauly (1980) encontró una relación entre $M$, los parámetros de crecimiento del modelo de von Bertalanffy ( $K$ y $L_{\infty}$ ) y la temperatura anual promedio del hábitat $\left(T,{ }^{\circ} \mathrm{C}\right)$ sobre la base de datos compilados de 175 stocks de peces. Para aplicar este método se consideraron los parámetros de ambos sexos (Tabla 2), y una temperatura promedio anual de $8^{\circ} \mathrm{C}$. Sin embargo, en el proceso de estimación de varianza, se consideró una distribución uniforme para la temperatura, entre un valor mínimo de $3^{\circ} \mathrm{C}$ y un máximo de $10^{\circ} \mathrm{C}$.

Hoening (1983) encontró una relación empírica entre la tasa de mortalidad total ( $Z$ ) y la edad máxima observada $\left(T_{\max }\right)$ de varias especies de peces, moluscos y cetáceos (Tabla 1). Muchos de los datos pertenecen a stocks no explotados o levemente explotados de tal manera que $\mathrm{Z} \approx M$. Una alternativa a $T_{\max }$ que representa la edad máxima observada, es contar con un estimador de la longevidad máxima esperada utilizando el método de Taylor (1960), quien supone que la edad máxima teórica $\left(t_{M}\right)$ ocurre cuando una cohorte alcanza el 95\% de la longitud asintótica, i.e.

$$
t_{M}=t_{0}+\frac{3}{K}
$$

donde $t_{0}$ y $K$ son parámetros de crecimiento del modelo de von Bertalanffy. En este trabajo, se utilizó $t_{M}$ como estimador de $T_{\text {max }}$.

\section{Tabla 2}

Parámetros de crecimiento en longitud para ambos sexos comunicados para $D$. eleginoides

\begin{tabular}{|c|c|c|c|c|c|}
\hline Océano & Area & $\begin{array}{c}L_{\infty} \\
(\mathrm{cm})\end{array}$ & $\begin{array}{c}K \\
\left(\text { año }^{-1}\right)\end{array}$ & $\begin{array}{c}t_{0} \\
\text { (año) }\end{array}$ & Autor \\
\hline \multirow[t]{6}{*}{ Pacífico } & Chile centro-sur & 177,8 & 0,109 & 0,000 & Pavéz et al. (1983) \\
\hline & & 218,0 & 0,048 & $-0,664$ & Rubilar et al. (1999) \\
\hline & & 152,2 & 0,085 & $-0,590$ & Oyarzún et al. (2003a) \\
\hline & & 176,2 & 0,058 & $-1,647$ & Oyarzún et al. (2003b) \\
\hline & Chile sur-austral & 210,8 & 0,064 & $-0,432$ & Aguayo \& Cid (1990) \\
\hline & & 212,6 & 0,066 & $-0,477$ & Young et al. (1992) \\
\hline \multirow[t]{5}{*}{ Atlántico } & Georgia del sur & 170,8 & 0,088 & 0,000 & CCAMLR (1995) \\
\hline & & 170,9 & 0,087 & 0,160 & Aguayo (1992) \\
\hline & & 204,0 & 0,056 & $-0,545$ & Zacharov \& Frolkina (1976) \\
\hline & & 175,0 & 0,071 & 0,005 & Shust et al. (1990) \\
\hline & & 207,0 & 0,075 & $-0,290$ & Cassia (1998) \\
\hline
\end{tabular}

Growth parameters in length for both sexes as communicated for $D$. eleginoides 
Alverson \& Carney (1975) plantean la siguiente ecuación para estimar la edad de máxima biomasa $\left(t^{*}\right)$ de una cohorte o edad crítica, i.e.

$$
t^{*}=t_{0}+\frac{1}{K} \ln \left[\frac{3 K}{M}+1\right]
$$

A partir de esta ecuación se puede obtener una expresión para estimar la tasa de mortalidad natural, i.e.

$$
M=\frac{3 K}{\left[\exp \left(K\left(t^{*}-t_{0}\right)\right)-1\right]}
$$

donde la constante 3 corresponde al exponente isométrico de la relación longitud-peso $(b=3) ; K$ y $t_{0}$ son parámetros del modelo de crecimiento de von Bertalanffy. Alverson \& Carney (1975) sugirieron utilizar la relación empírica $t^{*}=0,38 \times T_{\max }$, donde $T_{\max }$ corresponde a la edad máxima observada de la especie. En este trabajo se utiliza el enfoque de Cubillos (2003), el cual consiste en estimar el promedio de la razón entre la talla crítica $\left(L^{*}\right)$ y longitud asintótica $\left(L_{\infty}\right)$ a partir de un grupo de especies cercanas utilizando los datos $\left(L_{\infty}\right.$ $K$, y $M$ ). Para ello se utilizaron los datos comunicados por Pauly (1980), y se estimó la razón:

$$
\omega=\frac{L^{*}}{L_{\infty}}=\left(1-\exp \left(-K t^{*}\right)\right)
$$

En ausencia de datos de especies cercanas al bacalao de profundidad, se optó por utilizar el valor promedio de esta razón para 175 especies de peces, i.e. $\bar{\omega}=0,62$ $(\mathrm{CV}=0,22)$, y puede ser utilizado para determinar $M$ mediante la expresión reportada en la Tabla 1.

\section{Mortalidad natural edad-dependiente}

Chen \& Watanabe (1989) desarrollan una aproximación analítica para determinar la mortalidad natural por grupos de edad. Ellos dividen la mortalidad natural en tres fases: inicial, mortalidad estable, y mortalidad senescente. Estas fases se relacionan inversamente con tres fases de crecimiento: temprano, crecimiento estable, y senescente. De acuerdo con esta correspondencia, la mortalidad natural edad-específica, $M_{t}$, se asume inversamente proporcional al crecimiento, $G_{t}$, i.e.

$$
M_{t}=\frac{C}{G_{t}}
$$

donde $G(t)$ es representado por:

$$
G_{t}= \begin{cases}1-\exp \left(-K\left(t-t_{0}\right)\right), & t \leq t_{m} \\ a_{0}+a_{1}\left(t-t_{m}\right)+a_{2}\left(t-t_{m}\right)^{2}, & t \geq t_{m}\end{cases}
$$

donde:

$$
\left\{\begin{array}{l}
a_{0}=1-\exp \left(-K\left(t_{m}-t_{0}\right)\right) \\
a_{1}=K \exp \left(-K\left(t_{m}-t_{0}\right)\right) \\
a_{2}=-0,5 K^{2} \exp \left(-K\left(t_{m}-t_{0}\right)\right)
\end{array}\right.
$$

$\mathrm{y}$

$$
t_{m}=-(1 / K) \ln \left(1-\exp \left(K t_{0}\right)\right)+t_{0}
$$

donde $t_{m}$ representa la edad máxima de esperanza reproductiva, mientras que las constante $K$ y $t_{0}$ se refieren a parámetros del modelo de crecimiento de von Bertalanffy. En la sentencia inicial, la constante $C$ es una constante proporcional. Teóricamente, se puede considerar que $C=K$, y la estimación de la mortalidad natural por grupos de edad se reduce a las siguientes ecuaciones:

$$
M_{t}=\frac{K}{G_{t}}
$$

ó

$$
M_{t}= \begin{cases}K /\left(1-\exp \left(-K\left(t-t_{0}\right)\right)\right), & t \leq t_{m} \\ K /\left(a_{0}+a_{1}\left(t-t_{m}\right)+a_{a}\left(t-t_{m}\right)^{2}\right), & t \geq t_{m}\end{cases}
$$

Esta es la ecuación fundamental para estimar la mortalidad natural edad-específica. Esta ecuación se utilizó para analizar valores más probables de $M$, promediando $M_{t}$ entre un rango de edades en que se pueda asumir que permanece constante y que podría corresponder a la fracción explotada del recurso, lo que según Arana et al. (1994) comienza a partir del grupo de 8 años de edad. Se utilizaron los parámetros de crecimiento en longitud de ambos sexos de $D$. eleginoides (Tabla 2), para obtener estimaciones edadespecífica de $M$ promedio y sus respectivos intervalos de confianza.

\section{Procedimiento para estimar la varianza}

De acuerdo con Cubillos et al. (1999), el procedimiento sugerido aquí es un tipo de remuestreo bootstrap paramétrico de Efron (1985), que se evalúa numéricamente por medio del enfoque Monte Carlo. 
Para este procedimiento, un número de valores alternativos e igualmente probables de $M\left(M_{R}\right)$ se generan usando una forma paramétrica de la distribución del error, donde $R=1,2, \ldots, R_{\max }$, siendo $R_{\max }$ un número grande $(\geq 1000)$. En este estudio se consideró $R_{\max }=3000$ para cada modelo empírico. La estimación de la varianza asociada con este bootstrap paramétrico (BP) es entonces:

$$
V \hat{A} R_{B P}(M)=\frac{\sum_{R=1}^{R \max }\left(M_{R}-\bar{M}_{B P}\right)^{2}}{R_{\max }-1}
$$

donde

$$
\bar{M}_{B P}=\frac{\sum_{R=1}^{R_{\max }} M_{R}}{R_{\max }}
$$

Luego, los límites de confianza se obtienen con un método percentil, lo cual involucra ordenar el conjunto de valores alternativos de $M\left(M_{R}: R=1,2, \ldots, R_{\max }\right)$.

Para implementar el método de estimación de varianza, se analizó la correlación entre los parámetros de crecimiento y el error estándar de los mismos. Para ello, se estableció la correlación entre $K$ y el logaritmo natural de $L_{\infty}$ y entre $t_{0}$ y el logaritmo natural de $L_{\infty}$. Para incorporar la autocorrelación entre los parámetros se utilizó la siguiente aproximación lineal:

$$
Y_{i}=\bar{Y}+r_{y, x} \frac{s_{y}}{s_{x}}\left[X_{i}-\bar{X}\right]
$$

En la expresión (14) se toma en cuenta la correlación entre los parámetros alternativos e igualmente probables de $K$ y $t_{0}$, los que se hicieron depender del logaritmo de $L_{\infty}$ y del coeficiente de correlación. Los valores alternativos e igualmente probables del logaritmo de $L_{\infty}$ se realizó según:

$$
X_{R}^{*}=\bar{X}+s_{x} Z_{0,1}
$$

donde $X_{R} *$ son valores alternativos e igualmente probables de $X\left(=\ln L_{\infty}\right), \bar{X}$ es el promedio, $s_{x}$ es el error estándar del parámetro y $Z_{0,1}$ es una variable aleatoria distribuida normal con media cero y varianza igual a la unidad. Para transformar el logaritmo de la longitud asintótica a la escala aritmética, el antilogaritmo fue corregido por $\exp \left(s_{x}^{2} / 2\right)$, siendo $s_{x}$ el error estándar del parámetro en la escala logarítmica. Para el caso de la talla de primera madurez sexual se consideró una distribución uniforme, y también para el caso de la temperatura promedio del hábitat.

El error de predicción se incorporó según los supuestos de Cubillos et al. (1999) para los modelos empíricos utilizados por ellos. En el caso del modelo de Alverson \& Carney (1975), el error de predicción consistió en evaluar valores alternativos e igualmente probables para la razón $\omega$, considerando un $\mathrm{CV}=22 \%$ (Cubillos 2003).

\section{Resultados}

Los parámetros de crecimiento disponibles para ambos sexos de bacalao de profundidad (Tabla 2) están correlacionados (Tabla 3), lo que implica considerar esta estructura correlacionada en la evaluación de la precisión de los estimados de $M$.

El resumen estadístico de 3000 valores alternativos e igualmente probables de $M$, considerando tanto la incertidumbre en los parámetros de entrada como los errores de predicción en cada modelo empírico de estimación, se resumen en la Tabla 4 . Al considerar el error en los parámetros de entrada, se aprecia que el modelo de Rickhter \& Efanov (1976) genera el mayor coeficiente de variación $(\mathrm{CV}=25,2 \%)$, seguido por el modelo de Pauly (1980), el cual a su vez genera un estimado promedio de $M=0,10$ año $^{-1}$ con intervalos de confianza de 0,07 a 0,12. Los modelos más precisos fueron los de Hoening (1983) y Alverson \& Carney (1975).

\section{Tabla 3}

Valor promedio, error estándar y correlación de los parámetros de crecimiento en longitud disponibles para ambos sexos de $D$. eleginoides

Average, standard error and correlation values for the growth parameters in length available for both sexes of $D$.

eleginoides

\begin{tabular}{cccc}
\hline & Promedio & Error estándar & $\begin{array}{c}\text { Correlación } \\
\text { con } \ln \left(L_{\infty}\right)\end{array}$ \\
\hline $\ln \left(L_{\infty}\right)$ & 5,223 & 0,035 & 1,000 \\
$K$ & 0,073 & 0,006 & $-0,617$ \\
$\mathrm{t}_{0}$ & $-0,407$ & 0,099 & 0,129 \\
\hline
\end{tabular}


Tabla 4

Resumen estadístico de $\mathbf{3 0 0 0}$ valores alternativos e igualmente probables de mortalidad natural estimada con a) sólo error en los parámetros de entrada, y b) considerando el error en los parámetros de entrada y error de predicción simultáneamente para D. eleginoides

Statistics summary of 3000 equally likely alternative values of the natural mortality rate, which have been estimated with: a) input error in parameters, and b) by considering both input error and prediction error simultaneously for $D$. eleginoides

\begin{tabular}{lcccc}
\hline \multicolumn{4}{c}{ Modelos empíricos } \\
\cline { 2 - 5 } & Pauly (1980) & $\begin{array}{c}\text { Rickhter \& } \\
\text { Evanof (1976) }\end{array}$ & Hoening (1983) & $\begin{array}{c}\text { Alverson \& } \\
\text { Carney (1975) }\end{array}$ \\
\hline \multicolumn{3}{l}{ a) Sólo error en los parámetros de entrada: } & & \\
\hline Promedio & 0,10 & 0,14 & 0,11 & 0,13 \\
DS & 0,02 & 0,04 & 0,01 & 0,01 \\
CV (\%) & 15,58 & 25,15 & 5,16 & 5,23 \\
Mediana & 0,10 & 0,14 & 0,11 & 0,13 \\
Linf 95\% & 0,07 & 0,09 & 0,10 & 0,12 \\
Lmax 95\% & 0,12 & 0,21 & 0,12 & 0,15 \\
\hline
\end{tabular}

b) Con error en los parámetros de entrada y error de predicción:

\begin{tabular}{lrrrr}
\hline Promedio & 0,11 & 0,15 & 0,12 & 0,15 \\
DS & 0,05 & 0,07 & 0,05 & 0,09 \\
CV (\%) & 47,62 & 48,13 & 40,88 & 60,19 \\
Mediana & 0,10 & 0,14 & 0,11 & 0,13 \\
Linf95\% & 0,04 & 0,04 & 0,06 & 0,05 \\
Lmax95\% & 0,23 & 0,30 & 0,24 & 0,39 \\
\hline
\end{tabular}

Al incorporar el error de predicción se obtiene una alta incertidumbre en todos los modelos; esto es, coeficientes de variación del orden del 40,9 a 60,2\%. Las medidas de tendencia central, no obstante, tienden a mantenerse. La mediana es exactamente igual ya sea al incorporar o no el error de predicción, pero los límites de confianza son más amplios en relación con la incertidumbre que generan solamente los parámetros de entrada.

Se destaca que cuando se considera el error en los parámetros de entrada, la distribución de frecuencia de los valores alternativos e igualmente probables tiende a seguir la distribución del error de los parámetros de entrada. Por ejemplo, para el método de Rickhter \& Efanov (1976) se consideró una distribución uniforme para la talla de primera madurez sexual. Esta distribución uniforme se traspasa a los valores alternativos e igualmente probables de $M$ (Fig. 1), a pesar que la talla se transforma a edad con el error correlacionado de los parámetros de crecimiento. Sin embargo, al incorporar el error de predicción los 3000 valores de $M$ tienden a distribuirse normal (Fig. 1), mientras que en el caso de los modelos de Pauly (1980), Hoening (1983) y Alverson \& Carney (1975), aparece una distribución del tipo log-normal.

Las estimaciones de $M$ por grupos de edad con el método de Chen \& Watanabe (1989), se basaron sólo con los conjuntos de parámetros de crecimiento disponibles en que $t_{0}<0$, el promedio y los límites de confianza se presentan en la Fig. 2. El valor promedio de $M$ en el rango de edades 8 - 20 años fue estimada en 0,11 año $^{-1}$, valor que sería consistente con aquellos estimados por los modelos empíricos. 
Sin error de predicción
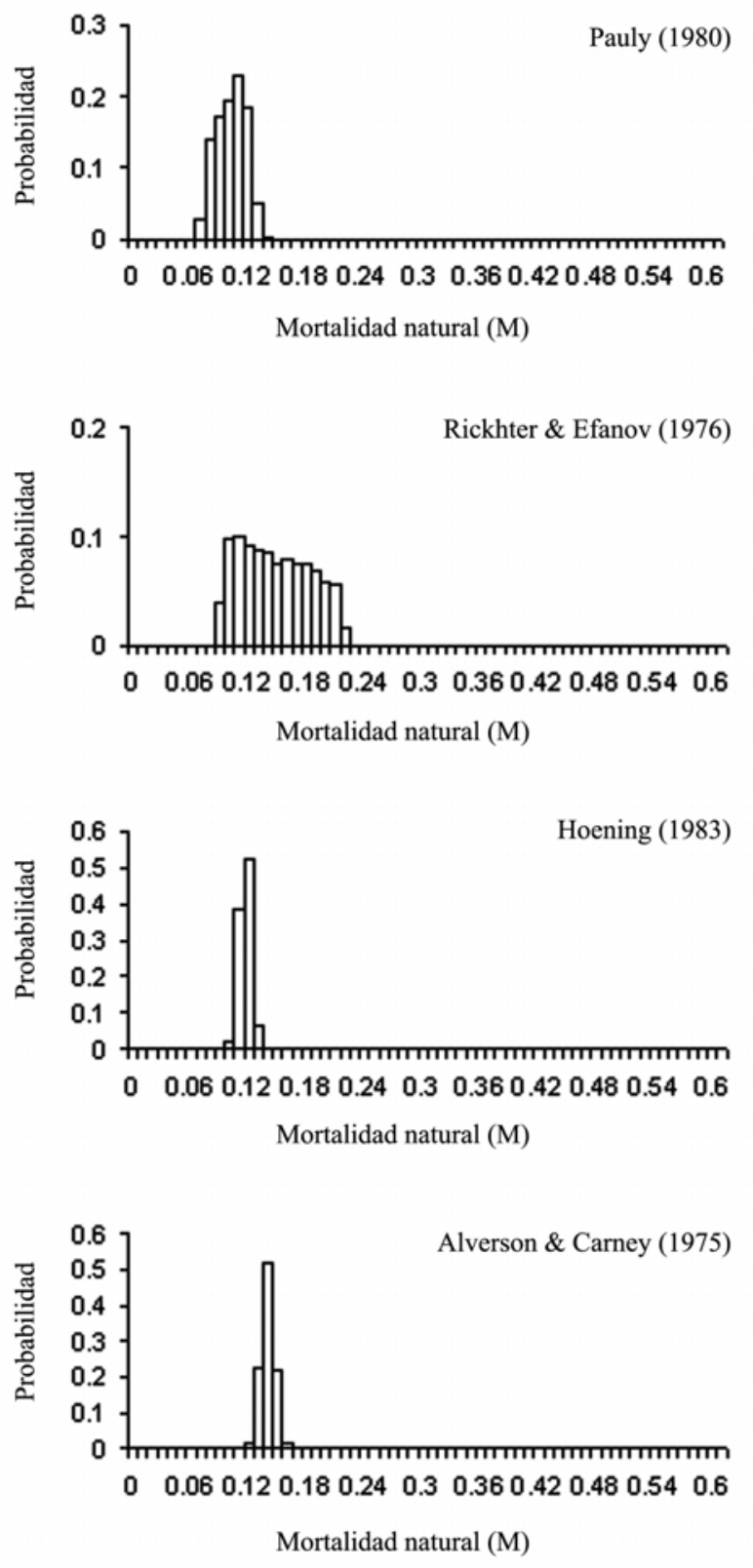

Con error de predicción
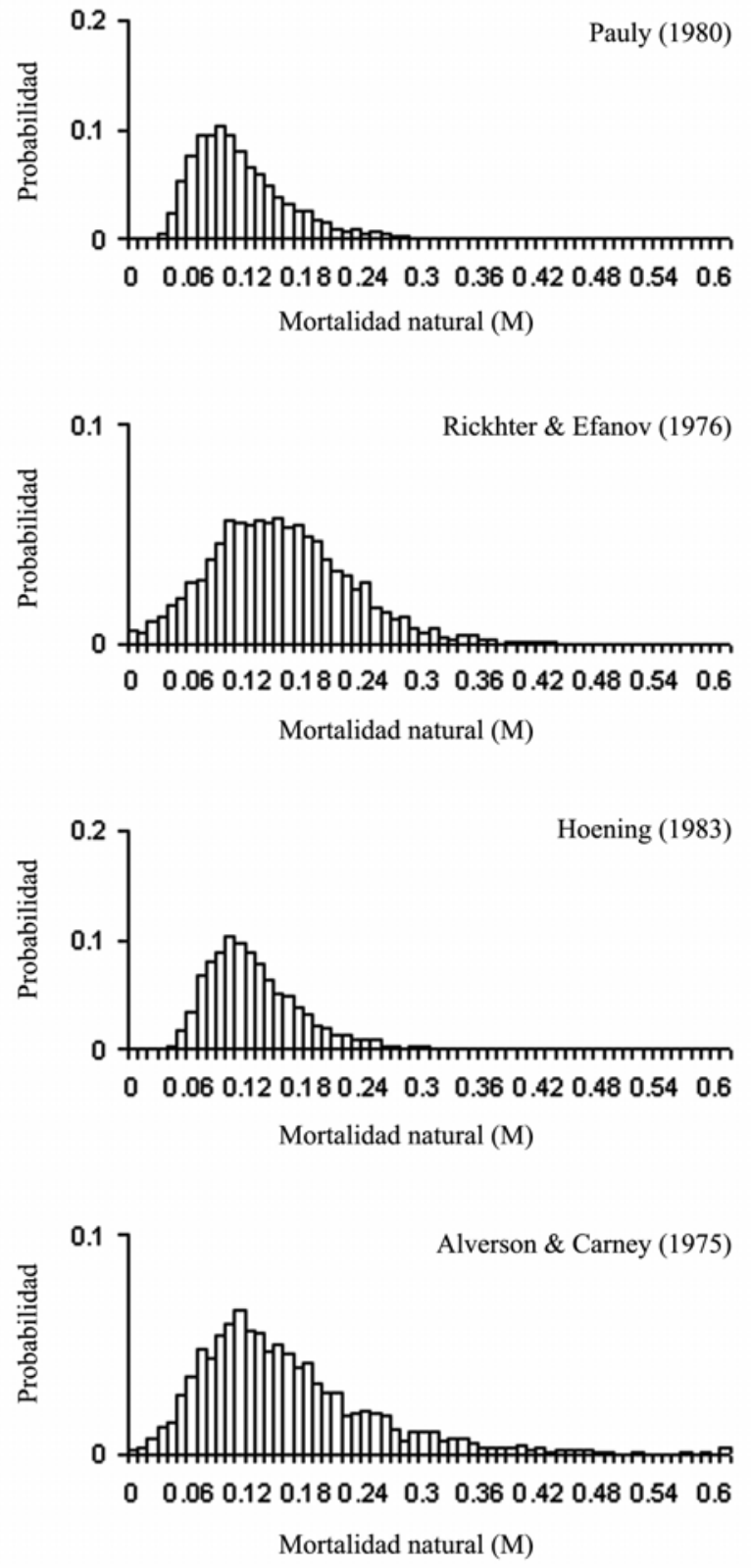

\section{Figura 1}

Distribución de probabilidad empírica de los 3000 valores alternativos e igualmente probables de mortalidad natural para $D$. eleginoides

Empirical probability distribution based on 3000 equally likely alternative values of the natural mortality rate for $D$. eleginoides 


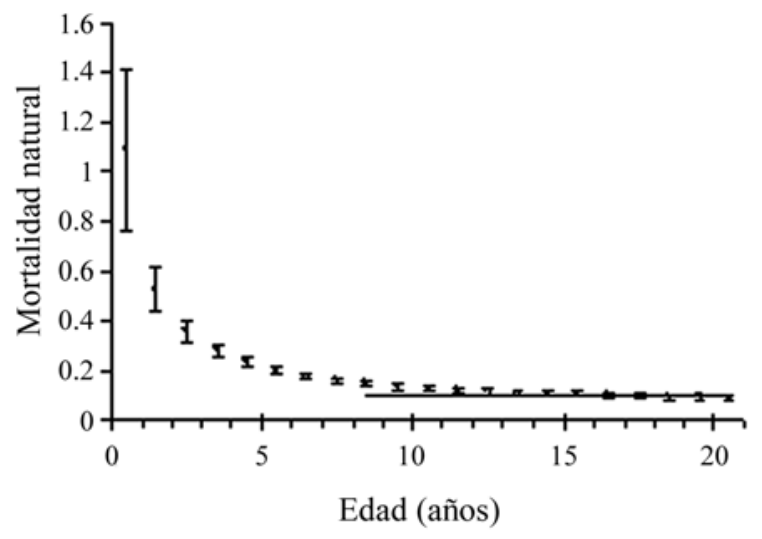

Figura 2

Estimación promedio de la mortalidad natural por clases de edad para $D$. eleginoides utilizando el método de Chen \& Watanabe (1989). La línea representa el valor promedio entre los 8 y 20 años de edad $\left(M=0,11\right.$ año $\left.^{-1}\right)$

Average estimates for the natural mortality rate per age classes of D. eleginoides through the method of Chen \& Watanabe (1989). The horizontal line represents the average value between 8 and 20 year of age $\left(M=0.11 \mathrm{yr}^{-1}\right)$

\section{Discusión}

Vetter (1988) indica que los métodos para estimar la tasa de mortalidad natural $(M)$ a partir de los parámetros de la historia de vida, presentan dos ventajas: (a) requieren una mínima cantidad de datos, y (b) son útiles en demostrar tendencias entre especies y en el desarrollo de la teoría ecológica. No obstante, ya que solamente estiman un único e impreciso valor de $M$, no serían eficientes desde el punto de vista de la precisión de $M$ ni para determinar la variabilidad de $M$ entre grupos de stocks. En efecto, las extrapolaciones no serán mejores que los métodos utilizados para estimar los valores de $M$ utilizados en las regresiones. De acuerdo con Cubillos et al. (1999), la precisión en las estimaciones de mortalidad natural utilizando simultáneamente el error de predicción y el de los parámetros de entrada utilizando modelos empíricos puede ser muy baja. En efecto, Pascual \& Iribarne (1993) evalúan el poder predictivo de los modelos empíricos más comunes utilizados para estimar $M$ y encuentran que los errores de predicción de las estimaciones de la mortalidad natural son importantes. Para el caso del bacalao de profundidad, lo anteriormente expuesto se corrobora ya que los coeficientes de variación fueron del orden de 41-60\%.
El método menos preciso fue el de Alverson \& Carney (1975), según el enfoque de Cubillos (2003), lo que probablemente se debe a que se usó un valor promedio de 175 stocks de peces para la razón longitud crítica:longitud asintótica $(\omega=0,62 ; \mathrm{CV}=22 \%)$. De esta manera, se desprende que al aplicar tal procedimiento es recomendable utilizar solo especies filogenéticamente cercanas. Los métodos de Rickhter \& Efanov (1976), Pauly (1980) y Hoening (1984) resultaron en coeficientes de variación en el rango 41 a $48 \%$, los que implica también una baja precisión.

No obstante lo anterior, una baja precisión no quiere decir que los métodos empíricos no posean exactitud, sino más bien que las estimaciones de $M$ con estos métodos pueden contener límites de confianza muy amplios. En este sentido, cualquier comparación con la tasa mortalidad total $(Z)$ para inferir la tasa de mortalidad por pesca que se haga, podría determinar que esta última pueda ser totalmente insignificante, o bien que en algunos casos $Z$ sea incluso menor que el valor de $M$ (Hall et al. 2004). En efecto, los límites de confianza superiores que se obtuvieron en este estudio indican que la mortalidad natural del bacalao de profundidad estaría en el rango $0,2-0,4$ año $^{-1}$, valores que representan mortalidades altas para una especie longeva y de madurez tardía. En este contexto, los valores de tendencia central deben manejarse con un decimal ya que no tiene sentido utilizar centésima para un parámetro poco preciso. En este sentido, al considerar que los valores medianos para el bacalao de profundidad fluctúan entre 0,1 y 0,14 año $^{-1}$, se recomienda utilizar $M=0,1$ año $^{-1}$ en modelos de evaluación de stock de este recurso. Esta recomendación coincide con el valor "más probable" sugerido por Arana et al. (1994) para el bacalao distribuido en torno a la isla Georgia del Sur.

El método de Chen \& Watanabe (1989) permite estimaciones de mortalidad dependientes de la edad. El método no ha sido muy aplicado en teleósteos para estimar $M$, sin embrago está siendo muy utilizando en rayas y tiburones debido a la carencia de relaciones empíricas para este grupo de peces (Cortés 1999, Cortés 2002, Carlson et al. 2003). Aunque el método es dependiente del valor del parámetro $t_{0}$ de la ecuación de crecimiento de von Bertalanffy, produce estimaciones en el orden de magnitud de los métodos empíricos. En el caso del bacalao de profundidad, las estimaciones de $M$ con este método fueron bastante altas en la fracción de peces menor a 4 años $\left(0,28\right.$ a 1,1 año $\left.^{-1}\right)$. Estas altas tasas de mortalidad en la fracción juvenil podrían estar 
indicando que el bacalao de profundidad sufre un alto impacto predatorio en la fracción juvenil. No obstante, aunque la disminución de la mortalidad con la edad es un proceso esperable en teleósteos (Beyer 1989, Caddy 1996, Abella et al. 1997), no se recomienda utilizar dichas estimaciones de $M$ por clases de edad en evaluación de stock hasta que no sean verificadas a través de estudios tróficos de los predadores del recurso.

La estimación promedio de $M$ obtenida para la fracción de edades comprendidas entre 8 y 20 años edad $\left(M=0,1\right.$ año $\left.^{-1}\right)$, fue consistente con las estimaciones medianas obtenidas con los modelos empíricos, y con los valores utilizados por varios autores en la evaluación del recurso (Shust et al. 1990, Arana et al. 1994, Des Clers et al. 1996, Zuleta et al. 1998). La tasa de mortalidad natural se estabiliza a partir del grupo de 8 años de edad, fracción que representa la fracción explotada según Arana et al. (1994). Se podría pensar que al utilizar los parámetros de crecimiento para estimar $M$ con modelos empíricos, se estarían tomando en cuenta todos los grupos de edad. Sin embargo, esto no es así, pues la tasa de mortalidad que se utiliza para construir los modelos empíricos usualmente proviene de la fracción explotable (e.g. curvas de pesca o la relación mortalidad total versus esfuerzo de pesca). En este contexto, se verifica con el modelo analítico de Chen \& Watanabe (1989) que la tasa de mortalidad natural en el bacalao de profundidad sería del orden de 0,1 año ${ }^{-1}$ en la fracción vulnerada por la pesquería.

Si bien es cierto en este estudio se recomienda utilizar $M=0,1$ año $^{-1}$ como valor de compromiso en evaluación de stock, la información contenida en este estudio en términos de la distribución de valores alternativos e igualmente probables (Tabla 4), podría permitir utilizar dichas distribuciones como conocimiento informativo a priori de $M$ en modelos de evaluación integrados bajo un enfoque bayesiano, tal que permitan estimar la distribución a posteriori (ver Hall et al. 2004). Alternativamente, la información generada en este estudio podría permitir restringir el espacio paramétrico de $M$ si se desea una estimación con métodos de evaluación estadísticos estructurados por edad con información auxiliar (e.g. Wang \& Liu 2006). En consecuencia, si bien la estimación de $M$ con modelos empíricos puede ser poco precisa, hoy en día se cuenta con herramientas que podrían estimar $M$ en modelos de evaluación de stock sin que sea necesario asumir un valor conocido y libre de error.

\section{Agradecimientos}

Los autores agradecen a C Oyarzún, S Gacitúa, y G Aedo (Universidad de Concepción), por los comentarios al manuscrito, así como a tres revisores anónimos cuyas sugerencias permitieron mejorar el manuscrito. Finalmente, se agradece el financiamiento otorgado por el Fondo de Investigación Pesquera a través del Proyecto FIP 2001-17.

\section{Literatura citada}

Abella AJ, JF Caddy \& F Serena. 1997. Do natural mortality and availability decline with age? An alternative yield paradigm for juvenile fisheries, illustrated by the hake Merluccius merluccius fishery in the Mediterranean. Aquatic Living Resources 10: 257-269.

Arana P, M Arredondo \& V Venturini. 1994. Pesca del bacalao de profundidad (Dissostichus eleginoides), efectuada por la flota chilena en torno a la isla Georgia del Sur (1991/1992). Investigaciones Marinas, Valparaíso 22: 67-84.

Aguayo, M. 1992. Preliminary analysis of growth of Dissostichus eleginoides from the Austral zone of Chile and South Georgia. CCAMLR Document WG-FSA 92/30: 1-8.

Alverson DL \& MJ Carney. 1975. A graphic review of the growth and decay of population cohorts. Journal du Conseil 36: 133-143.

Beverton RJH. 1963. Maturation, growth and mortality of clupeid and engraulid stocks in relation to fishing. Rapports et Procès-Verbaux des Réunions du Conseil Permanent International pour l'Exploration de la Mer 154: 44-67.

Beverton RJH \& SJ Holt. 1957. On the dynamics of exploited fish populations. UK Ministry Agriculture, Food and Fisheries, Fisheries Investigations (Ser. 2) 19: 1-533.

Beyer JE. 1989. Recruitment stability and survival-simple sizespecific theory with examples from the early life dynamics of marine fish. Dana 7: 45-147.

Caddy J. 1996. Modelling natural mortality with age in shortlived invertebrate population: definition of a strategy of gnomonic time division. Aquatic Living Resources 9: 197207.

Carlson JK, E Cortés \& DM Bethea. 2003. Life history and population dynamics of the finetooth shark (Carcharhinus isodon) in the northeastern Gulf of Mexico. Fishery Bulletin 101: 281-292.

Cassia MC. 1998. Comparison of age readings from scales and otoliths of the Patagonian toothfish (Dissostichus 
eleginoides) from South Georgia. CCAMLR Science 5: 191-203.

CCAMLR. 1995. Informe de la Decimocuarta Reunión del Comité Científico. Informes del Comité Científico SCCAMLR-XIV: $1-500$.

Chen S \& S Watanabe. 1989. Age dependence of natural mortality coefficient in fish population dynamics. Nippon Suisan Gakkaishi 55: 205-208.

Cortés E. 1999. A stochastic stage-based population model of the sandbar shark in the Western North Atlantic. En: Musick JA (ed). Life in the slow lane. Ecology and conservation of long-lived marine animals, pp. 115-136. American Fisheries Society Symposium 23, Bethesda, Maryland.

Cortés E. 2002. Incorporating uncertainty into demographic modeling: application to shark populations and their conservation. Conservation Biology 16: 1048-1062.

Cubillos LA, R Alarcón \& A Brante. 1999. Empirical estimates of natural mortality for the Chilean hake (Merluccius gayi): evaluation of precision. Fisheries Research 42: 147-153.

Cubillos LA 2003. An approximative relationship to estimate the natural mortality rate in fish stocks. Naga WorldFish Center Quarterly 26: 17-19.

Des Clers S, CP Nolan, R Baranowski \& J Pompert. 1996. Preliminary stock assessment of the Patagonian toothfish longline fishery around the Falkland Islands. Journal of Fish Biology 49(Suppl. A): 145-156.

Efron B. 1985. Bootstrap confidence intervals for a class of parametric problems. Biometrika 72: 45-48.

Hall NG, SA Hesp \& IC Potter. 2004. A Bayesian approach for overcoming incosistences in mortality estimates using, as an example, data for Acanthopagrus latus. Canadian Journal of Fisheries and Aquatic Science 61: 1202-1211.

Hoening JM. 1983. Empirical use of longevity data to estimate mortality rates. Fishery Bulletin 82: 898-902.

Moreno CA \& PS Rubilar. 1992. Notas sobre la mortalidad de Dissostichus eleginoides de la subarea 48.3. CCAMLR Selected Scientific Papers 9: 21-30.

Oyarzún C, S Gacitúa, M Araya, L Cubillos, M Salamanca, C Pino, R Galleguillos, G Aedo \& J Lamilla. 2003a. Asignación de edades y crecimiento de bacalao de profundidad. Informes Técnicos FIP-IT/2001-17: 1-74.
Oyarzún C, S Gacitúa, M Araya, L Cubillos, R Galleguillos, C Pino, G Aedo, M Salamanca, M Pedraza \& J Lamilla. 2003b. Monitoreo de la pesquería artesanal de bacalao de profundidad entre la VIII y IX Regiones. Informes Técnicos FIP-IT/2001-16: 1-98.

Paloheimo JE. 1980. Estimation of mortality rates in fish populations. Transactions of the American Fisheries Society 109: 378-386.

Pascual MA \& OO Iribarne. 1993. How good are empirical predictions of natural mortality? Fisheries Research 16: 1724.

Pauly D. 1980. On the interrelationships between natural mortality, growth parameters and mean environmental temperature in 175 fish stocks. Journal du Conseil 39: 175192.

Pavéz P, E Yañez, N Salas, W Tarky, P Rojas \& H Flores. 1983. Estudio del bacalao de profundidad Dissostichus amisus como recurso pesquero en la región de Valparaíso $\left(33^{\circ} \mathrm{S}-72^{\circ} \mathrm{W}\right)$. Evaluación biológica, tecnológica y económica. Estudios y Documentos Universidad Católica de Valparaíso 1/183: 1-160.

Ricker WE. 1975. Computation and interpretation of biological statistics of fish populations. Bulletin of the Fisheries Research Board of Canada 191: 1-382.

Rickhter VA \& VN Efanov. 1976. On one of the approaches to estimation of natural mortality of fish populations. International Commission of the Northwest Atlantic Fisheries, Research Document 76/VI/8: 1-12.

Robson DS \& DG Chapman. 1961. Catch curve and mortality rates from capture-recapture series. Special Publication of International Commission of the Northwest Atlantic Fisheries 4: 330-335.

Rubilar P, C Moreno \& A Zuleta. 1999. Edad y crecimiento de Dissostichus eleginoides (Pisces: Notheniidae) en la costa centro-sur de Chile (38 $19^{\prime}$ a $43^{\circ} \mathrm{S}$ ). Revista Chilena de Historia Natural 72: 285-296.

Shust RR, PS Gasiukov, RS Dorovkikh \& BA Kenzhin. 1990. The state of Dissostichus eleginoides. Stock and TAC for 1990/91 in Subarea 48.3 (South Georgia). CCAMLR WG-FSA-90/34: 1-27.

Taylor CC. 1960. Temperature, growth and mortality, the Pacific cockle. Journal du Conseil 26: 117-124.

Vetter EF. 1988. Estimation of natural mortality in fish stocks: a review. Fishery Bulletin 86: 25-43. 
Wang Y \& Q Liu. 2006. Estimation of natural mortality using statistical analysis of fisheries catch-at-age data. Fisheries Research 78: 342-351.

Yánez E \& L Pizarro. 1984. Análisis del rendimiento por recluta del bacalao de profundidad (Dissostichus amissus, Gill \& Towsend, 1901) en la región de Valparaíso (33ㅇ$72^{\circ} \mathrm{W}$ ). Investigaciones Marinas, Valparaíso 12: 87-92.

Young Z, A Zuleta, H Robotham, M Aguayo \& L Cid. 1992. Evaluación del stock de bacalao de profundidad entre las latitudes $47^{\circ}$ y $57^{\circ} \mathrm{S}$. Informe Técnico IFOPSUBPESCA: 1-63.

Young Z, H González \& P Gálvez. 1997. Análisis de la captura y del esfuerzo de pesca en la pesquería de bacalao de profundidad en la zona centro-sur. Informes Técnicos FIP-IT/96-32: 1-63.

Young Z, J Oliva, A Olivares \& E Díaz. 1999. Aspectos reproductivos del recurso bacalao de profundidad. Informes Técnicos FIP - IT/96-16: 1-101.
Zacharov GP \& ZA Frolkina. 1976. Some data on distribution and biology of the patagonian tothfish (Dissostichus eleginoides Smitt) occurring in the Southwest Atlantic (in Russian). Trudy AtlantNIRO 65: 143-150.

Zhivov VV \& Krivoruchko. 1990. On the biology of the Patagonian toothfish, Dissostichus eleginoides, of the Antarctic part of the Atlantic. Journal of Ichthyology 30: 142-146.

Zuleta A \& CA Moreno. 1992. Catch at age analysis applied to new fisheries: the case of Dissostichus eleginoides. CCAMLR Selected Scientific Papers. SC-CAMLRSSP/9: 3-9.

Zuleta A, C Moreno, P Rubilar \& J Guerra. 1998. Modelo de estrategias de explotación del bacalao de profundidad bajo incertidumbre del tamaño y rendimiento sustentable del stock. Informes Técnicos FIP, FIP - IT/96-41: 1-158.

Recibido el 16 de agosto de 2006 y aceptado el 31 de agosto de 2007 